\title{
A Novel HRBW-based approach to Mean shift algorithm for Target Tracking
}

\author{
XiaoWei Wang ${ }^{1, a}$, YaHui Han ${ }^{2, b}$, Antong Gao ${ }^{1, c}$ \\ ${ }^{1}$ Research Center for Unmanned Aerial Vehicle, Army Aviation Institute, Beijing 101123, China; \\ ${ }^{2}$ Mechanical Engineering Department, Army Aviation Institute, Beijing 101123, China \\ awangxiaoweis@163.com, ${ }^{b}$ han_huier@163.com, ${ }^{\text {cantong17@qq.com }}$
}

\begin{abstract}
Keywords: Target Tracking; Mean Shift; Background Weighted; Corrected Background Weighted; Histogram Ration; Histogram Ration Background Weighted

Abstract. To resolve the problem of localization errors in object tracking, which caused by the background pixels in an object model, a novel target tracking algorithm named Histogram Ration Background Weighted-based mean shift (HRBWBMS)is presented. In the proposed HRBWMS, unlike the standard mean shift, the target model is established based on object/background histogram log-likelihood ratio. A new weight transform method for target model based on object/background histogram log-likelihood ratio was introduced. The experimental results show that the proposed method not only accelerates the convergence, but also enhances anti-interference ability.
\end{abstract}

\section{Introduction}

Mean shift ${ }^{[1,2]}$ is widely used in various fields, such as object tracking, image segmentation, and pattern recognize. The central computational module of mean shift tracking algorithm is based on the mean shift iterations, and finds the most probable target position in the current frame. The color histogram is used to represent the target model and candidate target model. The Bhattacharyya coefficient is used as metric to calculate the dissimilarity between the target model and target candidate model. The selection of target feature is directly related to convergence speed and location accuracy.

However, in the traditional mean shift tracking, often the background information is included in the detected target region. If the correlation between target and background is high, the localization accuracy of object will be decreased. Comaniciu ${ }^{[3,4]}$ proposed the background-weighted histogram (BWH) algorithm in target model and target candidate model to reduce the interference of background in target localization. Jifeng Ning demonstrated the mean shift tracking with BWH algorithm was the same as the mean shift tracking with usual target represent because the BWH algorithm simultaneously decreased the probability of prominent background features in the target model and target candidate model ${ }^{[5]}$. Based on the mean shift iteration formula and background information exploiting, Jifeng Ning ${ }^{[6]}$ proposed the correct background weighted histogram mean shift (CBWHMS ). However, the CBWH-based method may not have the ability to locate moving object accurately from the same color background ${ }^{[7]}$. In this paper, the main aim is to improve the CBWHMS, using HRBW Scheme to reduce the interference of uniform background features in target localization.

The rest of the paper is organized as follows: Section 2 introduces the Histogram Ration Background Weighted-based mean shift algorithm. Section 3 present experiments to test the proposed HRBWMS method. Section 4 concludes the paper. 


\section{The novel HRBW-based approach to Mean shift algorithm for Target Tracking}

\section{The approved background-weighted histogram Scheme}

The target model's representation is directly related to convergence speed and location accuracy. So the idea of HRBWMS is to find features that best separate the tracked object from its immediate surroundings. The approved background-weighted histogram Scheme is to introduce the pixel weight based on object/background histogram log-likelihood ratio. The pixel weight is calculated as follows.

(1) First, target kernel histogram is established. The inner box in Fig. 1 is the object bounding box and the outer box represents the boundary of the local background.

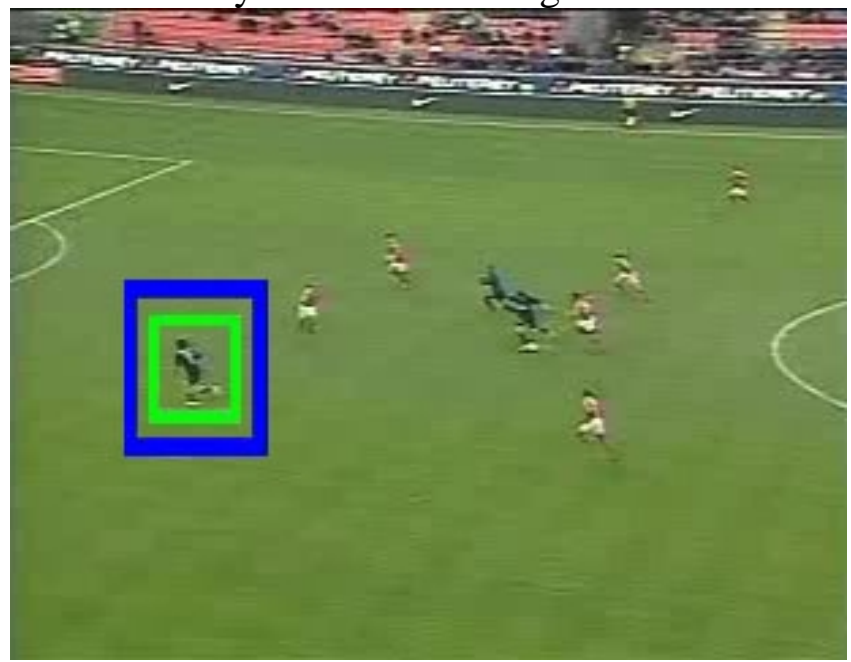

Fig. 1. Example of object and local background bounding boxes

The color histogram in inner box is used to represent the target. Denote by $\left\{x_{i}\right\}_{i=1, \mathrm{~L}, n}$ the pixels in the target region centered at $x_{0}$, which has $\mathrm{n}$ pixels, $h$ is the bandwidth. The probability of a feature $\mathrm{u}$, which is actually on of the m color histogram bins in the target model is derived by Eq. (1) that

$$
q(u)=\sum_{i=1}^{n} k\left(\left\|\frac{x-x_{0}}{h}\right\|^{2}\right) \delta\left[b\left(x_{i}\right)-u\right]
$$

Where $q(u)$ is the probability of the $\mathrm{u}^{\text {th }}$ element of target model, $\delta$ is the Kronecker delta function , $b\left(x_{i}\right)$ associates the pixel $x_{i}$ to the histogram bin, $k(x)$ is an isotropic kernel profile. We can form a probability distribution $g(u)$ by normalizing the histograms and dividing them by the normalized constant $C$

$$
g(u)=q(u) / C, \quad C=\frac{1}{\sum_{i=1}^{n} k\left(\left\|\frac{x-x_{0}}{h}\right\|^{2}\right) \delta\left[b\left(x_{i}\right)-u\right]}
$$

(2) The background color histogram is represented as $p$ and it is calculated by the surrounding area of the target.The background region is three times the size of the target as suggested in $^{[3]}$.The background model is

$$
p_{u}=\sum_{i=1}^{n_{k}} \delta\left[b\left(x_{i}\right)-u\right]
$$

Where $p(u)$ is the probability of the $u^{\text {th }}$ element of background model in ring area $n_{k}$ is the number of background pixels. We can form the normalized the background color histograms by dividing them into the number of pixels in the ring area

$$
b(u)=p(u) / n_{k}
$$


(3) The log likelihood of a feature value is given by

$$
L(u)=\log \frac{\max \{g(u), \delta\}}{\max \{b(u), \delta\}}
$$

Where $\delta$ is a small value that prevents division by zero or taking log of zero. The intuition behind the $\log$ likelihood is that it converts the multi-modal histograms to positive values for colors that correspond to the object and negative values for background colors and it will be close to zero for colors shared by both object and the background. The piecewise function curve is shown in Fig. 2 .

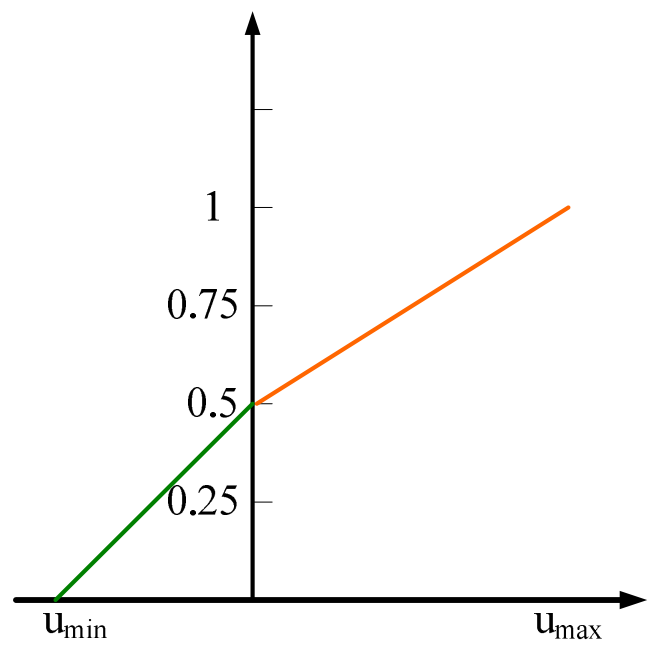

Fig.2. piecewise function curve

(4) Thus, we compute the transformed weight by the following equation:

$$
\mu(u)=\left\{\begin{array}{l}
0.5+\frac{L(u)}{L_{\max }(u)} \times 0.5, L(u)>0 \\
0.5, L\left(u_{1}\right)=0 \\
\frac{L_{\min }(u)-L(u)}{L_{\text {min }}(u)} \times 0.5, L(u)<0
\end{array}\right.
$$

$\mu(u)$ is lineal subsection function Whose value is between 0 and 1 .The transformation reduces the weights of those features belong to background and improves the weights of those color features belong to target. Where $L_{\max }$ is color feature which make $L(u)$ maximum, $L_{\min }$ is color feature which make $L(u)$ minimum。

The above HRBW uses normalization target kernel model and background color feature and forms the novel weight transform to target model. Then, HRBW is employed to transform only to target model but not target candidate model and is embed it into the mean shift tracking framework.

\section{The novel HRBW-based approach to mean shift algorithm}

The presented algorithm is summarized as follows.

(1)The HRBW is used to define a transformation to the representations of target model. Then the new target model is

$$
q_{u}=C \mu(u) \sum_{i=1}^{n} k\left(\left\|\frac{x_{i}-x_{0}}{h}\right\|^{2}\right) \delta\left[b\left(x_{i}\right)-u\right]
$$

Where $\mathrm{C}$ is the normalization constant

$$
C=1 / \mu(u) \sum_{i=1}^{n} k\left(\left\|\frac{x_{i}-x_{0}}{h}\right\|^{2}\right) \delta\left[b\left(x_{i}\right)-u\right]
$$


(2)In HRBW, Eq.(6) is employed to transform only the target model but not the target candidate model. That is to say, we reduce the prominent background features only in the target model but not in the target candidate model. So the new target candidate model is

$$
p_{u}=C_{h} \sum_{i=1}^{n_{k}} k\left(\left\|\frac{x_{i}-y}{h}\right\|^{2}\right) \delta\left[b\left(x_{i}\right)-u\right]
$$

Where $C_{h}=1 / \sum_{i=1}^{n} k\left(\left\|\frac{x_{i}-y}{h}\right\|^{2}\right) \delta\left[b\left(x_{i}\right)-u\right]$.

(3) a metric based on the Bhattacharyya coefficient is defined between the two normalized histograms $q_{u}$ and $p_{u}(y)$ as follows

$$
\rho(y)=\rho\left(q_{u}, p_{u}(y)\right)=\sum_{u=1}^{m} \sqrt{q_{u} p_{u}(y)}
$$

(4) Then we compute an offset from the current location $y$ to a new location $y_{1}$ according to the mean shift iteration equation

$$
\begin{gathered}
y_{1}^{\prime}=\frac{\sum_{i=1}^{n_{h}} x_{i} w_{i} g\left(\left\|\frac{x_{i}-y}{h}\right\|^{2}\right)}{\sum_{i=1}^{n_{h}} w_{i} g\left(\left\|\frac{x_{i}-y}{h}\right\|^{2}\right)} \\
w_{i}=\sum_{u=1}^{m} \sqrt{\frac{q_{u}}{p_{u}(y)}} \delta\left(b\left(x_{i}\right)-u\right)
\end{gathered}
$$

Where $g(x)$ is the shadow of the kernel profile $k(x): g(x)=-k^{\prime}(x)$.

\section{Experimental Results and discussions}

In this section, two representative experiments are performed to illustrate and testify the proposed HRBWMS in comparison with CBWHMS. The videos of different scenes, including the one that has uniform background colors, are used in evaluating the performance of the proposed algorithm. The two algorithms were implemented under the programming environment of Visual $\mathrm{C}++$.In all the experiments ,the RGB color model was used as the feature space and it was quantized into $16 \times 16 \times 16$ bins.Here we selected the Bhattacharyya kernel as recommended in so that $g(x)=-k^{\prime}(x)=1$

Experiment 1: The video sequences with spatial resolution $320 \times 240$ pixels are used to evaluate the proposed HRBW Histogram Scheme in comparison with the CBWH.Figure.1 show that the whole background is uniform green. The inner box in Fig. 1 is the object bounding box and the outer box represents the boundary of the local background. The background region is three times the size of the target. The target is initialized as a rectangular region of size $50 \times 50$ (external rectangle excluding the target region).Fig. 2(a) and (b) shows the weighted derived from CBWH and HRBW respectively. 


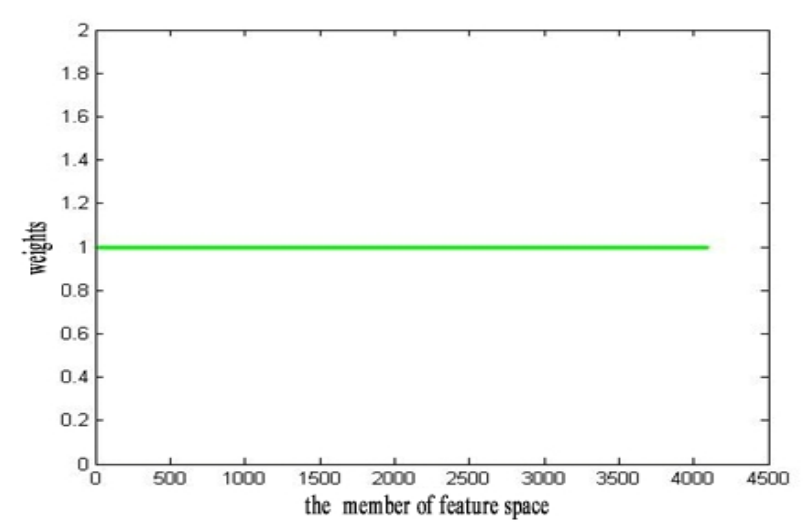

(a) the weighted derived from $\mathrm{CBWH}$

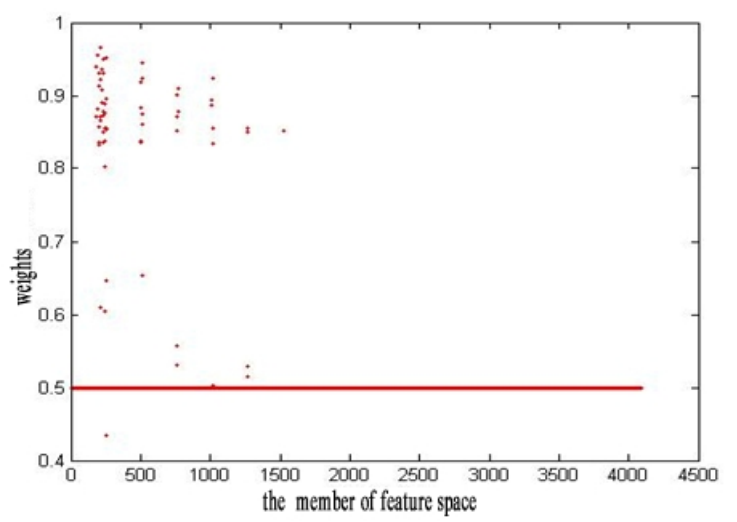

(b) the weighted derived from HRBW

Fig. 3. the weighted derived from $\mathrm{CBWH}$ and HRBW

As can be seen in Fig 3, the weights derived from CBWH are 1 when the background area is uniform color. So CBWH cannot effectively work. However, the HRBW transform scheme works much better in computing the weights when background is uniform.

Fig.4 illustrates the numbers of iterations by the CBWH and HRBW. The average number of iterations is 1.5507 for

CBWH and 0.6848 for HRBW. The color feature of target model is enhanced while the background features being suppressed in HRBW so that the mean shift tracking algorithm requires less computation and can more rapidly locate the

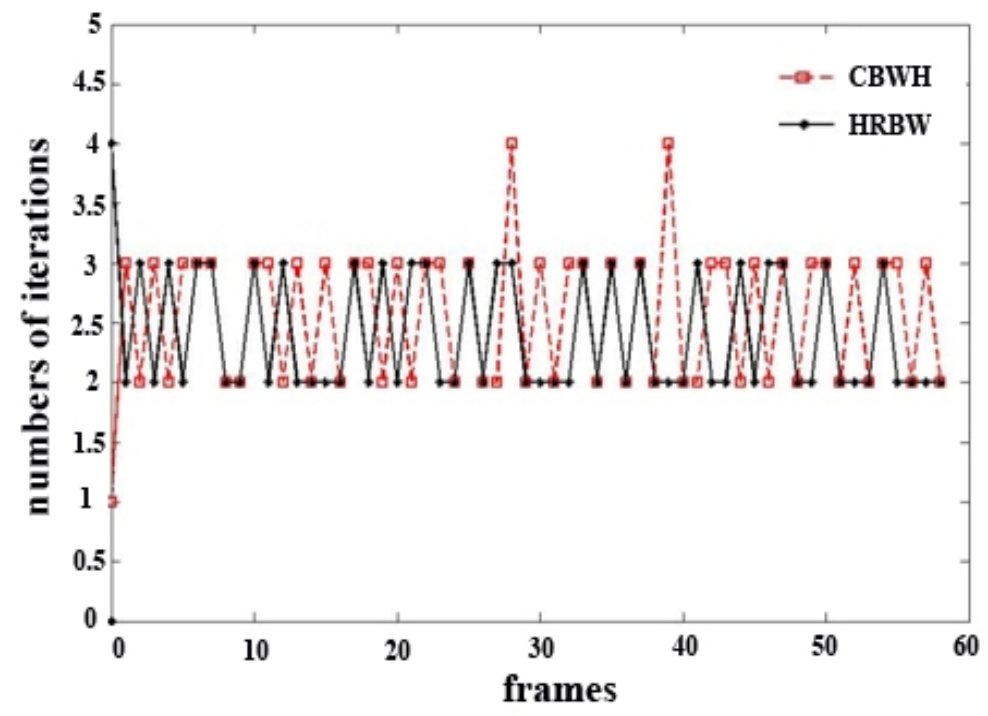

target.

Fig 


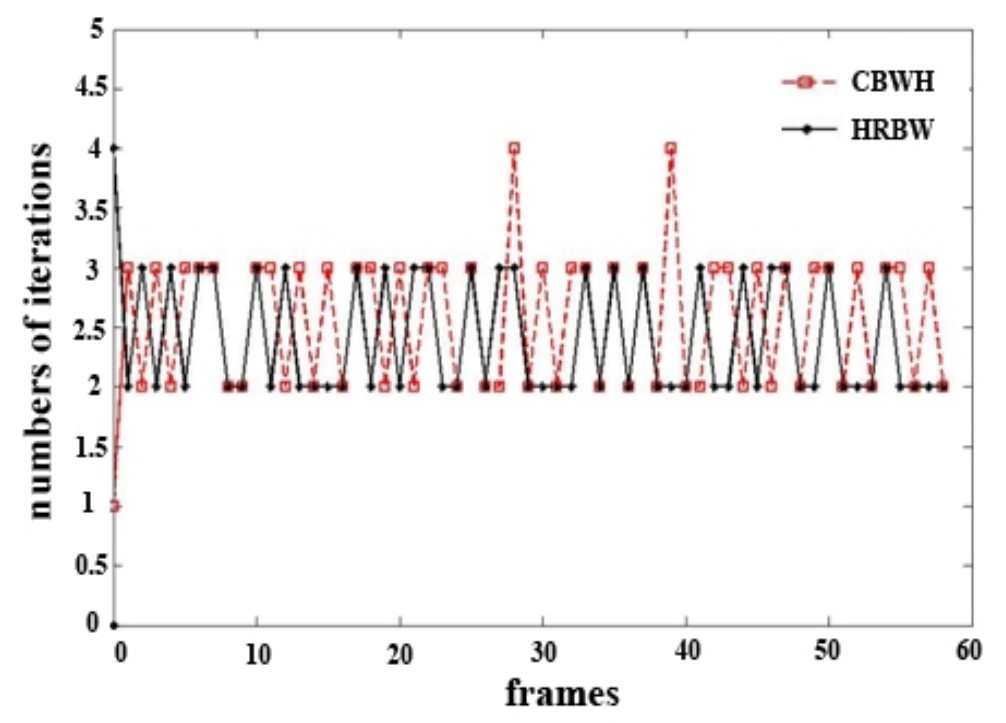

Fig.4 . number of iterations

The experiment result shows that the HRBW scheme which introduces object/background histogram log-likelihood ratio in the weight computation requires less computation and overcomes defects of CBWH in uniform background. Subsequently the mean shift tracking algorithm based HRBW is tested.

Experiment 2: The second experiment is on the sequence of passerby with partial occlusion. We use this sequence to test the robustness of the proposed HRBWMS to partial occlusion. This sequence has 719 frames of spatial resolution $352 \times 240$. The window size of target model is $27 \times 109$. The background model was initialized to be a region of size $55 \times 219$. The frames 1,6,47,183,232,350,526,578,645 are displayed. Experiment results in Fig.5 show that HRBHMS successfully tracks the passerby over the whole sequence with partial occlusion. Bhattchryya coefficients are shown in left up in per frame.

When track beginning, the target model is similar to the target candidate model, Bhattacharyya coefficients is greater than 0.8 .The Bhattacharyya coefficients is $0.935894,0.873408$ and 0.821130 respectively in 6,47,183frames. When target is partial occluded in 232,350,526frames, the Bhattacharyya is smaller, such as $0.710104,0.775150,0.650505$.However the mean shift tracking can track target continuously.

\section{Conclusions}

To resolve the problem that the background pixels in an object model induce localization errors in object tracking, a new target model establishing method based on HRBW is put forward. The new transform weight to target model based on object/background histogram log-likelihood ratio was introduced in the kernel histogram by using effectively normalization target kernel model and background color feature. The above HRBW Histogram Scheme reduces the weights of those features belong to background and improves the weights of those color features belong to target. Subsequently, this new weighted transform function is embedded into the mean shift tracking framework. Thus a mean shift tracking algorithm based HRBW is formed which transform only the target model but not the target candidate model. Experimental results indicate that the proposed method performs much better than $\mathrm{CBWH}$, especially in uniform background. 


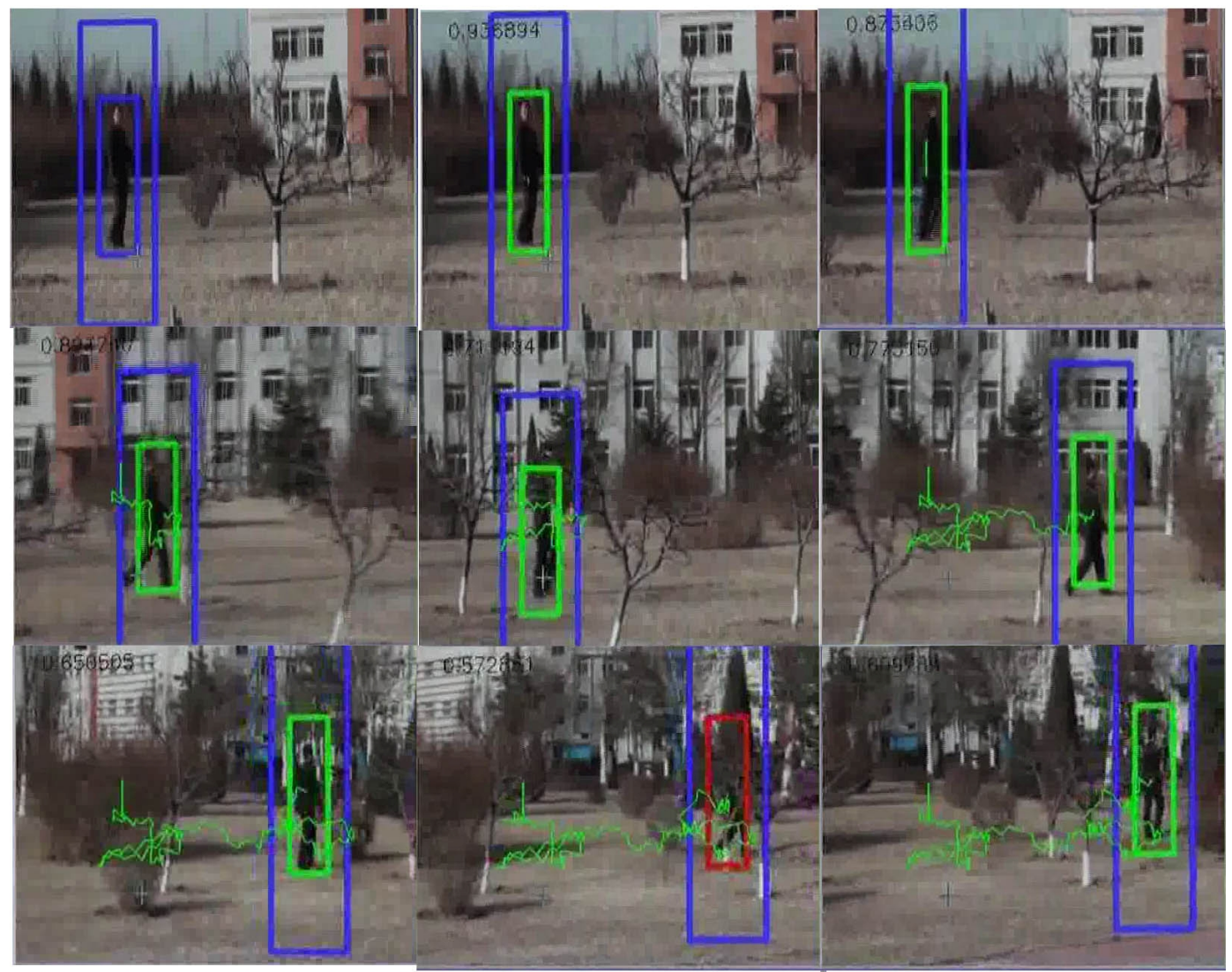

Fig.5. the tracking results on partial occlusion sequences

\section{References}

[1] K.Fukunaga, L.D.Hostetler. 'The estimation of the gradient of a density function, with applications in pattern recognition', IEEE Transaction Information Theory,21(1), pp.32 40 (1975).

[2] D. Comaniciu, V.Ramesh, P. Meer. 'Kernel based object tracking',IEEE transactions on Pattern Analysis and Machine Intelligence, 25(4),pp564-575(2003).

[3] RT. Collins, Yanxi Liu,M.Leordeanu.'Online selection of discriminative tracking features',IEEE transaction on Pattern Analysis and Machine Intelligence, 27 (10),pp. 1631 1643(2005).

[4] D. Comaniciu, V.Ramesh,'Mean Shift and optimal prediction for efficient object tracking', Proceedings of the IEEE International Conference on Image Processing ,pp.70 73(2000).

[5] Jifeng Ning, Lei Zhang,David Zhang,et.al.'Robust Mean Shift tracking with corrected backgroundweighted histogram'.IET Computer Vision, 6(1) ,pp. 62-69(2012).

[6] Jifeng Ning. Image Segmentation and Object Tracking in Computer Vision.Xi'dian University Doctor parper (2009). ( In Chinese)

[7] Yao Zhao, Faliang Chang. The comparative study on histogram computation methods. Computing Technology and Automation , 26(10),pp.71-74(2007).( In Chinese) 